\title{
Eficiencia de las ayudas al desarrollo: El caso español
}

\author{
Martín-Pérez, Víctor \\ Martín-Cruz, Natalia** \\ Serrano-Rodríguez, María Josét
}

\section{Resumen}

En un mundo de recursos escasos, el sector de ayuda al desarrollo no sólo tiene que ser eficaz sino eficiente. El objetivo del presente trabajo es evaluar la eficiencia de los principales instrumentos de cooperación internacional al desarrollo, a través de los cuales se materializa la ayuda oficial al desarrollo bilateral española. Sobre una muestra de 72 instrumentos de cooperación internacional al desarrollo en cuatro países prioritarios de dos grandes áreas geográficas para la ayuda española (Ecuador, Guatemala, Marruecos, Mozambique), se analiza su eficiencia por medio del Análisis Envolvente de Datos, tomando en consideración los objetivos logrados y los recursos utilizados. Como muestran los resultados obtenidos, entre los instrumentos evaluados, las ayudas de convocatoria abierta permanente son los más eficientes. Se puede concluir que, conocer los resultados alcanzados por cada instrumento, permite a los financiadores plantear acciones correctoras y servir como criterio de selección ex-ante de futuros instrumentos.

Palabras clave: Cooperación internacional; análisis envolvente de datos; eficiencia; organizaciones no gubernamentales; desarrollo

Recibido: 15.01.13. Aceptado: 9.12 .14

* Doctor en Administración y Dirección de Empresas, Profesor Contratado Doctor, Departamento de Organización de Empresas y Comercialización e Investigación de Mercados, Facultad de Ciencias Económicas y Empresariales, Universidad de Valladolid, España. E-mail: vmartin@ eco.uva.es

** Doctora en Administración y Dirección de Empresas, Profesora Titular, Departamento de Organización de Empresas y Comercialización e Investigación de Mercados, Facultad de Ciencias Económicas y Empresariales, Universidad de Valladolid (España). E-mail: ambiela@ eco.uva.es

*** Doctoranda en Administración y Dirección de Empresas, Departamento de Organización de Empresas y Comercialización e Investigación de Mercados, Facultad de Ciencias Económicas y Empresariales, Universidad de Valladolid (España). E-mail: marjoserrano@gmail.com 


\title{
Development aids efficiency. The Spanish case
}

\begin{abstract}
In a world of scarce resources, the aid development sector not only has to be effective buy efficient. The aim of the present study is to evaluate the main instruments of international development cooperation efficiency through which the official bilateral Spanish development aid materializes. Upon a 72 instruments of international development cooperation sample in four priority countries from two big geographic areas for the Spanish aid (Ecuador, Guatemala, Marruecos, Mozambique), its efficiency is analyzed by the Data Envelopment Analysis, considering the achieved objectives and the resources used. As the obtained results show, between the evaluated instruments the permanent open call aids are the most efficient. It can be concluded that knowing each instruments achieved results allows the funders to present corrective actions and to serve as a selection criteria for future instruments.
\end{abstract}

Key Words: International cooperation; Data Envelopment Analysis; efficiency; nongovernmental organizations; development.

\section{Introducción}

Desde 1960, año en el que distintos países comenzaron a crear sus agencias especializadas en cooperación al desarrollo para diseñar e implementar los programas de ayuda oficial al desarrollo, la cuestión de la eficacia de la ayuda internacional sigue siendo un tema pendiente de resolver. Una frase contenida en el Informe de Desarrollo Humano del Programa de Desarrollo de las Naciones Unidas (2005) resulta ilustrativa de este hecho: "La ayuda al desarrollo es una de las armas más potentes en la guerra contra la pobreza. En el momento presente, esta arma está infrautilizada y mal enfocada. Hay muy poca ayuda y, gran parte de la que se proporciona, está escasamente relacionada con el desarrollo humano".

Si la eficacia de la ayuda al desarrollo se encuentra en una situación mejorable, en términos de eficiencia se puede decir que es un campo prác- ticamente inexplorado y ello por dos razones. En primer lugar, por el cuestionamiento realizado por los propios donantes y entidades componentes del sector no lucrativo de si merece la pena dedicar recursos a esta labor detrayéndolos de las actuaciones directas sobre los beneficiarios de la ayuda. En segundo lugar, por la ausencia de una metodología de medición acorde a la complejidad del desafío planteado.

El objetivo de este trabajo es evaluar la eficiencia de diferentes Instrumentos de Cooperación Internacional al Desarrollo (ICID) utilizados por la Agencia Española de Cooperación Internacional para el Desarrollo (AECID), en concreto, ayudas de convocatoria abierta y permanente frente a subvenciones a Organizaciones no Gubernamentales para el Desarrollo (ONGD). Los instrumentos corresponden al período 2002-20061, ejecutados en cuatro países objetivo prioritario de la ayuda 
española al desarrollo: Ecuador, Guatemala, Marruecos y Mozambique.

La evaluación de la eficiencia se lleva a cabo por medio del Análisis Envolvente de Datos (DEA, por las siglas en inglés de Data Envelopment Analysis), que permite evaluar de forma objetiva y numérica, la eficiencia relativa teniendo en cuenta diversos inputs y outputs sin necesidad de asumir ponderaciones ni forma funcional de la función de producción, haciéndolo un método especialmente indicado para el sector no lucrativo y, en consecuencia, para la evaluación de los instrumentos de cooperación.

Hasta donde se tiene conocimiento, este es el primer trabajo que aplica la metodología DEA a la evaluación de ICID, superando las limitaciones de otras técnicas hasta ahora empleadas para valorar la eficiencia. Representa, por lo tanto, una contribución significativa a la literatura existente.

\section{Antecedentes teóricos de la ayuda oficial al desarrollo}

\author{
Numerosos estudios centrados \\ en los patrones de ayuda bilateral
}

(McKinley y Little, 1977 y 1979; Maizels y Nissanke, 1984; Dowling y Hiemenz, 1985; Boone, 1996; Svensson, 1999; Neumayer, 2003a y 2003b) ponen de manifiesto como los flujos de ayuda reflejan, principalmente, los intereses, tanto estratégicos como económicos, de los donantes en lugar de las necesidades de los beneficiarios. El interés nacional suele ser el motivo más destacado para los donantes en la concesión de ayuda bilateral, como prueba el hecho del mayor apoyo dado por los donantes a países con los que mantienen fuertes lazos económicos, culturales, políticos o estratégicos (Ram, 2003).

En una línea similar, trabajos como los de Alesina y Dollar (2000) o Kuziemko y Werker (2006) muestran como los vínculos coloniales o el apoyo a los países donantes en las votaciones en las Naciones Unidas incrementan la cantidad de ayuda recibida por un determinado país.

Abundando en el razonamiento anterior, los países donantes suelen ser reticentes a la hora de dirigir la ayuda hacia países de elevada población, confirmando este sesgo en la asignación

1 En el año 2001 se modificó el Plan Director de la Cooperación Española sentándose las bases de los instrumentos actualmente utilizados para canalizar la ayuda española al desarrollo de carácter bilateral no reembolsable. Estos instrumentos dan prioridad a las ONGD como actores ejecutores de esa ayuda en lugar de ser directamente implementada, en la mayor parte de los casos, por la AECID como se venía haciendo hasta ese momento. Además, es un periodo de fuerte crecimiento en el volumen de recursos para tratar de dar cumplimiento al objetivo de dedicar un $0,7 \%$ del PIB a la ayuda al desarrollo. A partir del año 2007 el volumen de fondos dedicados por España a la ayuda al desarrollo no ha dejado de descender, consecuencia de la crisis económica sufrida por el país y de las dificultades presupuestarias experimentadas, dando lugar a una caída alarmante del número de instrumentos financiados, actuaciones realizadas y países beneficiarios. El resultado es que estos años constituyen un periodo "anormal" para ser analizado porque la ayuda española al desarrollo se encuentra muy lejos de su objetivo razonable y, además, su motivación ha sido estrictamente presupuestaria dejando de lado la mayor parte de directrices, referencia en las agencias de cooperación de otros países, que la habían venido guiando. 
Eficiencia de las ayudas al desarrollo: El caso español

Martín-Pérez, Víctor; Martín-Cruz, Natalia y Serrano-Rodríguez, María José

por numerosas evidencias empíricas (Isenman, 1976; Dowling y Hiemenz, 1985; Trumbull y Wall, 1994; Wall, 1995; Alesina y Dollar, 2000; Bandyopadhyay y Wall, 2007). Las razones ofrecidas, según Younas (2008), son el menor impacto marginal de la ayuda según aumenta la población, la carencia mostrada por los países más poblados tanto de la experiencia como del conocimiento administrativo necesario para absorber grandes cantidades de ayuda y, quizá el más importante, para los donantes es relativamente más fácil ejercer influencia política sobre países pequeños que sobre países grandes.

En un intento por mejorar el impacto de la ayuda, la Declaración de París (OCDE, 2005) propone establecer indicadores para llevar a cabo su cuantificación. No obstante, el desarrollo de indicadores genera controversia porque, tal y como sugiere Boone (1996), la eficacia de la ayuda al desarrollo no debería medirse por su impacto en términos de crecimiento del Producto Interior Bruto (PIB), como ha venido siendo habitual, sino por reducción de pobreza y fomento del desarrollo humano y económico.

Con todo, desde la Declaración de París (OCDE, 2005) se observa un creciente interés en los donantes por alcanzar los objetivos de desarrollo del milenio, como demuestran las declaraciones de Accra y de Busan (OCDE, 2008a y 2011). Para ello, la valoración de la eficacia de su ayuda debería tener en consideración si los flujos de ayuda logran un impacto positivo en los indicadores de desarrollo humano seleccionados.

Gran parte de la evaluación viene aún guiada por los donantes y está diseñada para satisfacer las necesidades de los financiadores externos (O’Brien, 2009). En el caso de los grandes donantes públicos como USAID, UKAID, JICA, AECID, SIDA, NORAD (Agencias de Cooperación Internacional para el Desarrollo de Estados Unidos, Reino Unido, Japón, España, Suecia y Noruega, respectivamente), los criterios de evaluación se fundamentan en los principios para la evaluación de la ayuda al desarrollo del Comité de Ayuda al Desarrollo de la Organización para la Cooperación y el Desarrollo Económico (OCDE-CAD), basados en la relevancia, impacto, eficacia y sostenibilidad, a través de los cuales se trata de valorar si los proyectos de cooperación realizados son útiles o no y si deben mantenerse en el futuro.

Aunque sea una mejora importante respecto a lo hecho en el pasado, en un mundo de recursos escasos, el sector de ayuda al desarrollo, además de ser eficaz (alcanzar los objetivos) debe, y esto es lo realmente importante, ser eficiente, es decir, qué nivel de recursos se utiliza para conseguir un determinado objetivo (Duflo y Kremer, 2003; Banerjee, 2009). Ser más eficiente supone poder llevar a cabo más actuaciones con la misma cantidad de recursos, permitiendo no sólo multiplicar el efecto de los recursos destinados a ayuda al desarrollo sino también rebajar la presión sobre los organismos e instituciones donantes.

\section{El análisis de la eficiencia}

El propósito de este trabajo consiste en evaluar la eficiencia de Ios ICID, por lo tanto, es necesario, en primer lugar, determinar qué se entiende por eficiencia. Es ampliamente aceptado el concepto de eficiencia como relación entre el nivel de objetivos alcanzados 
y el volumen de recursos utilizados (Hernangómez-Barahona et al, 2007). Aquí se plantea un primer problema como es, dada una determinada relación entre objetivos y recursos, cómo saber si es eficiente o, dicho de otra forma, establecer un estándar adecuado. Un segundo problema viene dado por las múltiples dimensiones que puede tener el objetivo (outputs) y los distintos recursos empleados en su obtención (inputs), haciendo necesario valorar todas las dimensiones simultáneamente y asignar ponderaciones a los factores organizativos (Hernangómez-Barahona et al, 2009).

Con el fin de evaluar la eficiencia de los ICID, se debe definir su objetivo, para ello es preciso comprender tanto la estructura donde se integran como el marco de actuación. Estos proyectos forman parte de organizaciones cuyo plan de acción radica en intentar mejorar la calidad de vida en países en vías de desarrollo y la forma de realizarlo consiste en gestionar proyectos destinados a resolver una necesidad, o conjunto de necesidades, concreta. En consecuencia, si el objetivo de los ICID viene relacionado con la pregunta ¿qué se está intentando conseguir?, la respuesta obtenida sería mejorar la calidad de vida y generar bienestar en las personas en países en vías de desarrollo, objetivo compartido con el subsector formado por las ONGD globalmente consideradas.

En función de lo expuesto, el objetivo de los ICID puede formularse como generar bienestar a la mayor cantidad de personas posible y, la forma de lograr este objetivo es encargándose de cubrir necesidades concretas. Así, se puede refinar aún más la definición y plantear el objetivo como cubrir la necesidad específica, para la que fueron creados, al mayor número posible de personas.

Extendiendo este razonamiento a las entidades ejecutoras de los ICID, si existiera un mercado de ONGD, el donante valoraría más aquella organización con mayor capacidad para generar bienestar, o de generar bienestar a una mayor cantidad de personas, es decir, elegiría la organización más eficiente en la ejecución del instrumento de cooperación elegido. Por lo tanto, a la hora de evaluar la eficiencia de los ICID se pueden extrapolar los argumentos utilizados para valorar la eficiencia de operación de las ONGD.

Evaluar la eficiencia de entidades no lucrativas, como lo son las ONGD, y de los instrumentos utilizados por las mismas, en este caso los proyectos de cooperación para el desarrollo, presenta, como ya se expuso, ciertas dificultades al ser su objetivo más difícil de identificar y medir por comparación con las empresas $y$, además, tener múltiples dimensiones (calidad del proyecto, adecuación a las necesidades atendidas, sostenibilidad, impacto, entre otros), siendo la función de producción difícil de determinar.

Los métodos propuestos para evaluar la eficiencia de las ONGD se han basado en medidas contables, fundamentalmente, análisis de ratios. Entre éstas se pueden citar el precio, la ratio de proyectos, la eficiencia técnica y la eficiencia asignativa. El precio es definido como el costo que tiene para un donante adquirir una unidad monetaria de output para los beneficiarios de la entidad. Sin tener en cuenta la deducción fiscal, se mide como la inversa del porcentaje de gastos dedicados a proyectos (Weisbrod y Domínguez, 1986; Posnett y Sandler, 1989; Callen, 1994; Tinkelman, 1998). 
Eficiencia de las ayudas al desarrollo: El caso español

Martín-Pérez, Víctor; Martín-Cruz, Natalia y Serrano-Rodríguez, María José

La ratio de proyectos, definida como el porcentaje de los gastos totales correspondiente al gasto en proyectos, es utilizada por algunos autores (Baber et al, 2001; Roberts et al, 2004) como alternativa al precio, afirmando Baber et al, (2001) que esta medida puede indicar la estrategia de captación de fondos de la entidad no lucrativa.

Una medida muy similar, ampliamente utilizada, es la eficiencia asignativa (Callen y Falk, 1993; AndrésAlonso et al, 2006; HernangómezBarahona et al, 2009; Andrés-Alonso et al, 2010), calculada como la proporción representada por los gastos en proyectos sobre los ingresos totales de la entidad. La eficiencia técnica o ratio de gastos de administración (Callen y Falk, 1993; Greenlee y Brown, 1999; AndrésAlonso et al, 2006; HernangómezBarahona et al, 2009; Andrés-Alonso et al, 2010) viene definida como el porcentaje representado por los gastos de administración sobre los gastos totales.

Estas medidas no se corresponden con el concepto de eficiencia, relación entre objetivos alcanzados y recursos empleados, antes planteado, al no medir el grado de consecución de el/los objetivo/s, ni las cantidades de recursos consumidas, únicamente proporcionan información sobre el grado en el que una entidad no lucrativa dedica los recursos disponibles a su misión, definición de eficiencia establecida por Parsons (2003). Es decir, muestra el porcentaje medio de cada donación transferido por la organización a sus beneficiarios.

A pesar de sus limitaciones, estos indicadores, especialmente la eficiencia técnica y la asignativa, por su simplicidad, facilidad para obtener la información y posibilidades que dan de realizar comparaciones homogéneas entre entidades, son muy utilizados, tanto por los donantes (Hyndman, 1991; Khumawala y Gordon, 1997), a la hora de decidir a qué organizaciones realizar su donación, como por las propias entidades, para transmitir al conjunto de la sociedad una imagen de transparencia y cumplimiento de su misión, cuyo resultado sea una mayor captación de fondos.

Frente a los métodos contables cobran importancia los denominados métodos de frontera, técnicas a través de las cuales se puede medir la eficiencia relativa de un conjunto de organizaciones mediante la construcción de fronteras eficientes, subyaciendo bajo este concepto la noción de un nivel máximo alcanzable. El cálculo de la frontera eficiente se puede llevar a cabo utilizando métodos tanto paramétricos como no paramétricos.

Las técnicas paramétricas requieren especificar una forma funcional de la función de producción para poder estimar, por medio de una regresión, la máxima relación entre outputs e inputs de las unidades de la muestra. Estos métodos son muy sensibles a la especificación de la función de producción; si la forma funcional no está determinada correctamente se introducen importantes sesgos en los resultados por lo que, si no se puede llegar a una especificación cercana a la tecnología de producción subyacente, no se recomienda su uso.

Dentro de los métodos no paramétricos, la técnica fundamental es el Análisis Envolvente de Datos (DEA). EI DEA mide la eficiencia relativa de unidades homogéneas a través de la construcción de una frontera eficiente calculada sobre una medida global que relaciona objetivos (outputs) y los recursos empleados para obtener 
esos objetivos (inputs), permitiendo utilizar un gran número de variables y relaciones (restricciones).

Este método resulta especialmente indicado para el sector no lucrativo, tanto por adecuarse a las características singulares de las organizaciones participantes, dificultad de conocer los precios del producto/servicio generado, su función de producción o el hecho de trabajar con múltiples inputs y outputs, como por establecer una medida objetiva de la eficiencia en conjuntos de organizaciones carentes de indicadores agregados que den cabida a los múltiples objetivos simultáneamente perseguidos (Hernangómez-Barahona et al, 2009).

\section{La metodología DEA}

El Análisis Envolvente de Datos (DEA), desarrollado por Charnes et al (1978), consiste en una técnica de programación lineal que permite evaluar la eficiencia relativa de distintas unidades. El método identifica las unidades eficientes ${ }^{2}$ y construye una frontera formada por las combinaciones lineales entre dichas unidades. La eficiencia del resto de las unidades se mide conforme a su distancia con la frontera eficiente calculada.

La formalización del análisis se realiza como un modelo de optimización matemática cuyas variables a estimar son los índices de eficiencia, definidos como el cociente entre las sumas ponderadas de los outputs y las sumas ponderadas de los inputs. Es una técnica no paramétrica al no especificar una forma funcional concreta entre el máximo de outputs alcanzable y los inputs requeridos.

La aplicación de esta metodología permite obtener la medida de eficiencia para cada entidad, cuyos valores pueden variar entre 0 y 1 (donde 1 corresponde a la ubicación sobre la frontera eficiente), y determina las ponderaciones de forma endógena correspondiendo a los valores de mayor eficiencia posible. Por lo tanto, esta metodología permite realizar comparaciones entre entidades de forma objetiva, según su nivel de eficiencia, salvándose el problema de la comparación planteado con las medidas contables.

Existen diferentes versiones del DEA según los supuestos asumidos sobre la tecnología de producción y las restricciones incorporadas. La formulación matemática del modelo básico, suponiendo rendimientos constantes a escala, para la orientación output e input, se presenta en la Tabla 1 , donde $\phi_{0}$ y $\varphi_{0}$ representan los índices de eficiencia con cada una de las orientaciones, $y_{r j}$ y $x_{i j}$ representan las cantidades de output $r$ y de input $i$ de la entidad j, uro y vio representan las ponderaciones del output $r$ y del input $\mathrm{i}$, finalmente $\varepsilon$ representa un número positivo suficientemente pequeño por debajo del cual no pueden estar los ponderadores (Martínez, 2003).

2 Se sigue el criterio de eficiencia paretiana: una unidad es eficiente si ninguna otra produce más de algún output sin producir menos de algún otro y sin utilizar más de alguno de los inputs; o, si ninguna unidad produce la misma cantidad de outputs empleando menos de algún input y no más de los restantes (Martínez, 2003). 


\section{Tabla 1}

\section{Modelo de rendimientos constantes a escala (Modelo CCR)}

\begin{tabular}{|l|l|}
\hline Orientación output & Orientación input \\
Max $\quad \phi_{0}=\sum_{r=1}^{s} u_{r 0} y_{r 0}$ & Min $\quad \varphi_{0}=\sum_{i=1}^{m} v_{i 0} x_{i 0}$ \\
& \\
s.a. $\quad \sum_{r=1}^{s} u_{r 0} y_{r j}-\sum_{i=1}^{m} v_{i 0} x_{i j} \leq 0 ; \quad j=0,1, \ldots, n$ & s.a. $\quad \sum_{r=1}^{s} u_{r 0} y_{r j}-\sum_{i=1}^{m} v_{i 0} x_{i j} \leq 0 ; \quad j=0,1, \ldots, n$ \\
& \\
$\sum_{i=1}^{m} v_{i 0} x_{i 0}=1$ & $\sum_{r=1}^{s} u_{r 0} y_{r 0}=1$ \\
$u_{r 0} \geq \varepsilon \quad r=1,2, \ldots, s$ & $u_{r 0} \geq \varepsilon \quad r=1,2, \ldots, s$ \\
$v_{i 0} \geq \varepsilon \quad i=1,2, \ldots, m$ & $v_{i 0} \geq \varepsilon \quad i=1,2, \ldots, m$
\end{tabular}

Fuente: Martínez (2003)

Como se mencionó anteriormente, es posible incluir múltiples outputs e inputs en el análisis, sin embargo, según aumenta la cantidad de variables incluidas en el cálculo disminuye la capacidad de discriminación del $D E A^{3}$. Adicionalmente, es preciso recordar la no existencia de tests para determinar la significación estadística de las variables incluidas y evaluar la bondad del ajuste. Debido a estas consideraciones, como recomendación general, la suma de inputs y outputs no debe exceder un tercio del tamaño de la muestra (Martínez, 2003). Por otro lado, al evaluarse la eficiencia en términos relativos y ser esta técnica sensible a valores extremos, las unidades incluidas en el análisis deben ser homogéneas, es decir, aplicar la misma tecnología de producción y operar bajo un marco institucional idéntico.

Una última consideración viene dada por la elección de la orientación del análisis (output o input). Esta opción depende de la naturaleza del problema y se relaciona con el control de las entidades sobre las variables, si las unidades consideradas están restringidas en cuanto al manejo de inputs se opta por una orientación output y viceversa. En la orientación output una unidad es eficiente si ninguna otra puede producir mayor nivel de outputs con igual nivel de inputs. Por su parte, en la orientación input una unidad es eficiente

3 Una mayor cantidad de variables en relación con el número de observaciones de la muestra aumenta la probabilidad de que las unidades resulten eficientes en alguna de las variables. 
si no existe otra que disminuyendo la cantidad de inputs (manteniendo la proporción) consiga obtener la misma cantidad de outputs.

En este trabajo se utilizará el DEA para evaluar la eficiencia en proyectos de cooperación para el desarrollo dado que permite medir la eficiencia de acuerdo a la definición utilizada (relación entre objetivo y recursos) e incluir múltiples inputs y outputs sin realizar una especificación de la función de producción. Por otro lado, es posible realizar comparaciones válidas entre proyectos y la información requerida por el método se adapta a la información disponible para realizar el análisis.

La motivación fundamental de los trabajos que han aplicado el DEA en el sector no lucrativo viene dada por el deseo por valorar la eficiencia de las organizaciones no lucrativas en la utilización de sus recursos en la producción de los bienes y servicios generados, tomando en consideración los múltiples objetivos simultáneos perseguidos. La aplicación de esta metodología resulta de especial interés por cuanto permite identificar la reducción en los inputs necesaria para lograr la eficiencia (McMillan y Datta, 1998) así como los niveles de ineficiencia de las diferentes unidades (Farrell, 1957; Farrell y Fieldhouse, 1962; Charnes et al, 1994) y analizar sus causas.

La literatura respecto del estudio de la eficiencia en el sector no lucrativo es bastante abundante en los sectores de salud (Ley, 1991; Valdmanis, 1992; Magnussen, 1996; Al-Shammari, 1999;
García et al, 1999; Hofmarcher et al, 2002; Watcharasriroj y Tang, 2004) y educación (Johnes y Johnes, 1995; McMillan y Datta, 1998; Ruggiero, 1999; Avkiran, 2001; Korhonen et al, 2001; Martínez, 2003; Banker et al, 2004).

En el sector de la cooperación internacional para el desarrollo su aplicación es aún incipiente. No obstante, se han realizado trabajos que, utilizando la metodología DEA, intentan medir la eficiencia relativa de las ONGD (Martín-Pérez et al, 2005; HernangómezBarahona et al, 2006, 2007 y 2009), la eficiencia de las dos etapas del proceso productivo de las ONGD (Marcuello, 1999; García y Marcuello, 2007) o la eficiencia con que se ejecutan los proyectos de cooperación internacional al desarrollo (Martín-Cruz et al, 2012).

Si bien para la realización del DEA, tal y como se ha expuesto, no es necesario establecer una forma funcional de la función de producción ${ }^{4}$, si requiere determinar los outputs e inputs relevantes de la actividad que se esté evaluando, habiéndose empleado distintos mecanismos para su determinación, fundamentalmente, elaborar una lista de outputs e inputs, ya sea por observación de las unidades evaluadas o a partir de una revisión de la literatura. Posteriormente, se seleccionan aquellos considerados más pertinentes manteniendo la capacidad de discriminación del DEA (Marcuello, 1999; Martín-Pérez et al, 2005; Hernangómez-Barahona et al, 2006, 2007 y 2009; García y Marcuello, 2007; Martín-Cruz et al, 2012).

4 Se ha caracterizado una función de producción con rendimientos variables a escala a fin de separar la eficiencia técnica pura y la eficiencia de escala de las unidades productivas (Banker et al, 1984). 
Eficiencia de las ayudas al desarrollo: El caso español Martín-Pérez, Víctor; Martín-Cruz, Natalia y Serrano-Rodríguez, María José

Para determinar los outputs, es preciso determinar con anterioridad los objetivos a alcanzar. En este sentido, a nivel global, partiendo de la misión declarada por estas organizaciones (transformación del orden internacional) se han definido dos objetivos prioritarios, como son la erradicación de la pobreza en el Tercer Mundo y la educación para el desarrollo en las sociedades del Norte. En línea con estos objetivos, los trabajos que han aplicado el DEA en el sector de la cooperación internacional al desarrollo han utilizado como outputs los gastos en proyectos (Marcuello, 1999), volumen de ingresos captados (García y Marcuello, 2007) o el número de proyectos (Hernangómez-Barahona et al, 2006, 2007 y 2009; Martín-Pérez et al, 2005).

En cuanto a los inputs, se han medido a través de parámetros tales como gastos de funcionamiento, donaciones y subvenciones (Marcuello, 1999), número de voluntarios (Marcuello, 1999, García y Marcuello, 2007), gastos de administración (García y Marcuello, 2007), volumen de ingresos (García y Marcuello, 2007; HernangómezBarahona et al, 2006, 2007 y 2009; Martín-Pérez et al, 2005), número de empleados (García y Marcuello, 2007; Hernangómez-Barahona et al, 2006, 2007 y 2009; Martín-Pérez et al, 2005) o antigüedad de la organización (Hernangómez-Barahona et al, 2006, 2007 y 2009; Martín-Pérez et al, 2005).

Como puede observarse, hay algunos parámetros utilizados como inputs y también como outputs, aspecto

explicable por la estructuración en dos etapas del funcionamiento de las ONGD (acciones encaminadas a la captación de fondos en una primera etapa $y$ aplicación de los recursos obtenidos en la ejecución de proyectos en una segunda fase).

\section{La ayuda oficial al desarrollo: el caso español}

La Ayuda Oficial al Desarrollo (AOD) es definida por la OCDE (2008b) como las transferencias de recursos tanto a países receptores que figuran en las lista del Comité de Ayuda al Desarrollo $^{5}$ (CAD) como a instituciones multilaterales para el desarrollo. Esta ayuda es proporcionada por organismos oficiales con el objetivo de promocionar el desarrollo y bienestar de los países receptores. Adicionalmente, para ser caracterizadas como AOD, las referidas transferencias deberán ser de carácter concesional $^{6}$.

Dentro de la literatura de ayuda al desarrollo, las dos formas de implementación habitualmente consideradas son los flujos bilaterales de ayuda al desarrollo canalizados desde el gobierno donante al receptor y los proyectos ejecutados por ONGD en países en vías de desarrollo.

Estos últimos han adquirido mayor importancia (Meyer, 1995), tanto por el papel cada vez más importante desempeñado por las ONGD en el ámbito del desarrollo y el creciente porcentaje de ayuda al desarrollo por ellas canalizado, como por haber mostrado

5 En la lista del CAD se incluyen todos los países de ingresos medios y bajos, excepto los miembros del G8, Unión Europea y los países con fecha establecida para ingresar a la Unión Europea.

$6 \mathrm{Al}$ menos un $25 \%$ de la ayuda debe ser donación y no puede tener tasas mayores a las del mercado. 
una asignación de la ayuda siguiendo incentivos adecuados y una distribución directa a la población, evitando los peligros de una mala asignación y un mal empleo normalmente atribuidos a la ayuda al desarrollo bilateral (Jordan y Van Tuijl, 2006).

Como prueba de ello, todas las agencias de ayuda bilateral y las dos principales instituciones de ayuda multilateral (Banco Mundial y el Fondo Mundial de lucha contra el Sida, la Tuberculosis y la Malaria) prestan ayuda al desarrollo a través de ONGD, bien a través de proyectos propuestos y remitidos por las propias ONGD (como en Finlandia, Reino Unido, España o Irlanda, entre otros), por medio de acuerdos negociados para varios años con un conjunto de ONGD seleccionadas (Reino Unido, Irlanda, Finlandia, España, entre otros) o a través de los fondos asignados a las embajadas y oficinas país para que financien ONGD (Noruega, Suecia, Reino Unido o Finlandia, entre otros).

Los instrumentos de cooperación internacional al desarrollo, cuya eficiencia se pretende medir, forman parte de la AOD. Este trabajo se centra en instrumentos de AOD financiados por la AECID de tipo bilateral y de carácter no reembolsable, en línea con lo expuesto previamente para otras agencias de ayuda al desarrollo bilateral. Los principales instrumentos a disposición de la AECID (ver www.aecid. es), subvenciones a ONGD, y ayudas de convocatoria abierta y permanente, se explican a continuación.

Las Ayudas de Convocatoria Abierta y Permanente (CAP) tienen por objetivo la financiación de proyectos o actividades de cooperación al desarrollo de iniciativa particular. Las resoluciones de convocatorias indican las prioridades para la concesión de las subvenciones teniendo en cuenta el Plan Director de la Cooperación Española y el Plan Anual de Cooperación Internacional vigentes. Para la selección de las solicitudes presentadas se tienen en consideración los siguientes aspectos: la adecuación de las prioridades sectoriales y geográficas de la cooperación española; el grado de complementariedad con otras acciones relacionadas con la cooperación para el desarrollo; el contenido, relevancia y calidad de la actividad; la pertinencia y viabilidad de la actividad.

Las subvenciones a ONGD (SO) están motivadas por los principios de colaboración, complementariedad, calidad y eficacia de la ayuda. La AECID cuenta con dos instrumentos de financiación de la colaboración con estas organizaciones: convenios y proyectos. Los proyectos de cooperación al desarrollo tienen un único objetivo de desarrollo, en un solo país, para una población beneficiaria predefinida y sus efectos deben ser perdurables luego de finalizada la ejecución.

Por su parte, los convenios son de carácter plurianual y persiguen un objetivo general de desarrollo compartido entre la ONGD y la AECID. Puede tratarse de intervenciones en uno o más países e involucrar uno o más sectores. Tienen una duración máxima de cuatro años, prorrogable por 2 años adicionales. Para poder acceder a los convenios las ONGD deber ser previamente clasificadas por la AECID como "calificadas" sobre la base de criterios tales como años de experiencia, solvencia financiera, transparencia en la rendición de cuentas y medios humanos, entre otros.

La cooperación española establece prioridades geográficas para su ayuda en función de diversos factores 
Eficiencia de las ayudas al desarrollo: El caso español Martín-Pérez, Víctor; Martín-Cruz, Natalia y Serrano-Rodríguez, María José

(Plan Director de la Cooperación Española, 2010): indicadores de desarrollo, la presencia y articulación de la cooperación española en el país, el marco de asociación posible en el país, el potencial del país como socio de desarrollo y la posición relativa de la cooperación española respecto a otros donantes; estos criterios aplican desde el año 2001. Así, se definen tres grupos de países beneficiarios de esta ayuda.

El grupo A, denominado de asociación amplia, está formado por los países menos adelantados, de renta media-baja, con los cuales se puede establecer un marco de asociación a largo plazo, canalizar elevados volúmenes de AOD y utilizar diversos tipos de instrumentos.

El grupo B, de asociación focalizada, está integrado por los países menos adelantados y de renta mediabaja, con los cuales el programa de cooperación no permite una asociación del tipo A. En estos países el impacto en el desarrollo aumenta si la ayuda se enfoca en un aspecto determinado identificado en conjunto con el país socio. En este caso se realiza un uso más selectivo de instrumentos.

El grupo C, denominado de asociación para la consolidación de logros de desarrollo, el objetivo de la ayuda, en estos países, es la consolidación de políticas públicas inclusivas, la promoción de la cooperación sur-sur, la cooperación triangular y la provisión de bienes públicos globales.

Con respecto a la muestra y fuente de información, de un total de 34 países prioritarios en el primer Plan Director, de 19 países en el segundo Plan Director $y$, tras mantener reuniones con la Directora del Departamento de Ayuda Multilateral y ONGD, con el fin de conocer aquellos países donde se aplicaban diferentes instrumentos de AOD, con una percepción de eficiencia en su ejecución bastante diversa, cuatro países, Ecuador, Guatemala, Marruecos y Mozambique, fueron seleccionados. Todos ellos pertenecen al grupo A de prioridad geográfica mencionado en el epígrafe precedente.

Además, estos cuatro países responden a dos de los motivos esgrimidos por la literatura para que un país se convierta en receptor de ayuda, en el caso de Ecuador y Guatemala haber sido colonias españolas y mantener vínculos comunes (como lengua, cultura, estructuras institucionales), mientras en Marruecos y Mozambique priman los intereses estratégicos y económicos por el volumen de emigración recibido y los intensos intercambios comerciales mantenidos, siendo todos ellos países con no demasiada población.

El estudio abarca un intervalo de cinco años, desde 2002 al 2006, incluyendo los últimos datos disponibles cuando se llevó a cabo la recopilación de información (de junio a diciembre de 2009) y constituye un período lo suficientemente extenso para extraer conclusiones del análisis. La información, a falta de una base de datos, se obtuvo en la sede de la AECID utilizando, como fuente principal de información, los informes de las ONGD, de los técnicos de la AECID y de las auditoras de los proyectos ejecutados, asimismo, se llevaron a cabo entrevistas en profundidad a los técnicos de los países analizados.

En la Tabla 2 se presentan los valores referidos a la AOD bilateral recibida por estos países en los años 2002-2006, pudiendo observarse como el total de ayuda recibida por los países de la muestra, con la excepción del primer año, creció en el período 
de estudio, especialmente en el último año cuando se materializan las nuevas políticas de ayuda al desarrollo tendentes a alcanzar un porcentaje de un $0,7 \%$ del PIB. Asimismo, en relación con la participación que la ayuda a estos países representa sobre el total, el valor más bajo corresponde al año 2003 (7\%) mientras el valor más alto corresponde a 2006 (19\%), este último particularmente elevado explicado por un aumento significativo ese año de la ayuda bilateral a Guatemala.

\section{Tabla 2}

\section{Ayuda Oficial al Desarrollo bilateral española neta (en Euros)}

\begin{tabular}{lccccc}
\hline & 2002 & 2003 & 2004 & $2005^{\mathrm{a}}$ & \multicolumn{1}{c}{2006} \\
\hline Ecuador & 45.598 .265 & 21.798 .323 & 26.596 .134 & 38.816 .525 & 30.029 .369 \\
Guatemala & 18.577 .956 & 20.759 .018 & 17.992 .675 & 31.318 .118 & 178.292 .675 \\
Marruecos & 19.552 .778 & 13.565 .703 & 41.430 .125 & 23.356 .276 & 58.359 .434 \\
Mozambique & 35.586 .833 & 19.983 .881 & 26.233 .975 & 23.616 .859 & 26.739 .371 \\
TOTAL & 1.059 .188 .889 & 1.019 .099 .685 & 1.118 .180 .670 & 1.498 .725 .440 & 1.666 .671 .716 \\
\hline \multicolumn{7}{c}{ En porcentaje del total } \\
\hline Ecuador & 2002 & 2003 & 2004 & $2005^{\mathrm{a}}$ & 2006 \\
Guatemala & $4 \%$ & $2 \%$ & $2 \%$ & $3 \%$ & $2 \%$ \\
Marruecos & $2 \%$ & $2 \%$ & $2 \%$ & $2 \%$ & $11 \%$ \\
Mozambique & $3 \%$ & $1 \%$ & $4 \%$ & $2 \%$ & $4 \%$ \\
TOTAL & $11 \%$ & $2 \%$ & $2 \%$ & $2 \%$ & $2 \%$ \\
\hline a Los datos de & 2005 corresponden a la AOD bilateral bruta. & & \\
\hline
\end{tabular}

Fuente: Ministerio de Asuntos Exteriores y Cooperación (2009)

Sobre un total de 173 ICID ejecutados correspondientes a los cuatro países seleccionados durante los cinco años objeto de análisis, se pudo recabar información para 72 de ellos $(42 \%$ del total). Por tanto, la muestra se compone de 72 instrumentos de los cuales 20 son CAP y 52 corresponden a SO. Por otro lado, del total de 72 instrumentos, 13 corresponden a Ecuador, 9 a Guatemala, 30 a Marruecos y 20 a Mozambique. A diferencia de otros sectores de actividad, en el caso de los ICID no existe disponibilidad de una base de datos con información sobre su gestión, por lo tanto, en este trabajo se utiliza una base de datos propia, creada a partir de información proporcionada por la AECID, 
Eficiencia de las ayudas al desarrollo: El caso español

Martín-Pérez, Víctor; Martín-Cruz, Natalia y Serrano-Rodríguez, María José

en cuya realización se debieron resolver algunos problemas.

No existe un protocolo para la supervisión y control de estos instrumentos, consecuentemente, no se dispone de información cuantitativa para todos ellos y tampoco es posible obtener todos los ítems identificados para todas las unidades de la muestra; por otro lado, el período de ejecución de los instrumentos suele experimentar retrasos y extenderse sobre los plazos previstos. Esto implica que la muestra incluya instrumentos no culminados, lo que causa vacíos de información.

Adicionalmente, existen instrumentos vinculados: la AECID brinda subsidios vinculados a otros instrumen- tos específicos, o a un grupo de ellos, considerando estos subsidios como instrumentos independientes; no obstante, en la construcción de la base de datos fueron considerados como parte del instrumento al que están vinculados, al ser su objetivo facilitar la ejecución del mismo.

La AECID establece un conjunto de partidas contables para la elaboración de presupuestos de los ICID (cuadro 1). En la base de datos para la aplicación del DEA se estableció un paralelismo entre estas partidas y las del Plan Nacional de Contabilidad Pública, siendo estas últimas partidas las empleadas para identificar los inputs utilizados.

\section{Cuadro 1}

\section{Partidas contables para la elaboración de presupuestos de los Instrumentos de Cooperación Internacional al Desarrollo}

A I. Identificación/ A III Evaluación externa

A II. Auditorías

A IV. Total terrenos y/o inmuebles

A V. Construcción y reforma

A VI. Equipos, material y suministros

A VII. 1 Personal local

A VII. 2 Personal expatriado/ A VII. 3 Personal en sede

A VIII. Servicios técnicos

A VIIla. Sensibilización en España vinculada al proyecto

A X. Función terreno

A XI. Viajes, alojamientos y dietas

A XII. Gastos financieros

B I. Gastos administrativos de ONGD española

B I. Gastos administrativos de ONGD local

Fuente: Elaboración propia a partir del Plan General de Contabilidad Pública 
Las principales variables utilizadas para medir la eficiencia de las ONGD, conceptuando cuáles son sus objetivos y los principales recursos destinados a su consecución. Sin embargo, en este trabajo no se valora a las ONGD en su conjunto, si no a los instrumentos concretos empleados para operativizar su misión. Se hace necesario conceptuar cuál es el objetivo de estos instrumentos para poder establecer los outputs a medir y cuáles son los factores prioritariamente utilizados en su logro para, así, seleccionar los principales inputs.

Siguiendo a Martín-Cruz et al, (2012), el objetivo de los proyectos de cooperación internacional al desarrollo realizados por las ONGD es la maximización del "bienestar social" en la población donde se llevan a cabo. En línea con dicho trabajo, el objetivo planteado para los ICID es cubrir, al mayor número de personas posible, la necesidad específica para la que fueron creados estos instrumentos. Esto requiere establecer una lista de dimensiones para hacer operativo dicho objetivo, tales como número de actividades realizadas, número de familias atendidas, número de individuos beneficiados, número de áreas de intervención, número de entidades participantes o duración real ${ }^{7}$, para seleccionar aquellas relevantes con información disponible para un mayor número de instrumentos (Tabla 3 ).

A la hora de determinar cuáles serán las dimensiones utilizadas para medir el output se siguieron dos criterios de forma simultánea, la disponibilidad de información y el nivel de importancia concedido a estas dimensiones por los técnicos de la AECID. Se pretenden seleccionar dimensiones con información disponible para el mayor número de instrumentos posible, de modo que la comparación establecida por medio del DEA sea realmente significativa. Adicionalmente, se solicitó a los técnicos de la AECID encargados de la evaluación ex-ante, seguimiento, supervisión y evaluación ex-post de los diferentes instrumentos, como profundos conocedores de los mismos, valorasen la importancia de las diferentes dimensiones elegidas, empleando para ello una escala de seis niveles.

En la Tabla 3 se presentan los resultados obtenidos en ambos criterios. Las dos dimensiones más valoradas por los técnicos de la AECID, lamentablemente, son aquellas con un menor nivel de información disponible, por ello, el criterio finalmente aplicada para la selección de los outputs fue el de disponibilidad de datos.

7 La dimensión "duración real" merece ser explicada con más detalle. Generalmente, el tiempo invertido en un proceso productivo es tomado como un input y, en la comparación de procesos, se considera más eficiente el proceso con menor requerimiento de tiempo para igual nivel de coste. Sin embargo, en este caso, la duración se considera como un output, siendo la razón subyacente que un instrumento tendrá más capacidad de beneficiar a la población objetivo cuanto mayor sea el periodo de ejecución por estar más tiempo en contacto con ella. Por lo tanto, un donante enfrentado a la opción de financiar dos proyectos con igual coste preferirá aquel de mayor duración. 


\section{Tabla 3}

\section{Outputs para medir la eficiencia de los Instrumentos de Cooperación Internacional al Desarrollo}

\begin{tabular}{lcc}
\hline Concepto & $\begin{array}{c}\text { Disponibilidad } \\
\text { de datos }\end{array}$ & Orden de importancia \\
\hline Duración real en meses & $96 \%$ & 3 \\
Número de actividades & $95 \%$ & 6 \\
Número de áreas & $95 \%$ & 5 \\
Número de entidades & $84 \%$ & 4 \\
Número de individuos & $68 \%$ & 1 \\
Número de familias & $4 \%$ & 2 \\
\hline
\end{tabular}

Fuente: Martín-Cruz et al (2012)

En cuanto a los inputs, los principales factores empleados en el proceso productivo de los ICID se concretan, siguiendo a Martín-Cruz et al, (2012), en partidas tales como compras de materias primas, compras de otros aprovisionamientos, gastos de personal, dietas, servicios de profesionales independientes, reparaciones y conservación, arrendamientos y cánones, o material de oficina. En la Tabla 4 se resumen los principales inputs identificados para medir la eficiencia de los ICID.

\section{Tabla 4}

\section{Inputs para medir la eficiencia de los Instrumentos de Cooperación Internacional al Desarrollo}

\begin{tabular}{lcc}
\hline Concepto & $\begin{array}{c}\text { Instrumentos que } \\
\text { lo incluyen }\end{array}$ & $\begin{array}{c}\text { Importancia en } \\
\text { el gasto }\end{array}$ \\
\hline Compras & $97 \%$ & $45 \%$ \\
Sueldos y salarios & $93 \%$ & $34 \%$ \\
Comunicaciones y otros servicios & $91 \%$ & $12 \%$ \\
Servicios de profesionales independientes & $71 \%$ & $8 \%$ \\
Suministros & $71 \%$ & $2 \%$ \\
Reparaciones y conservación & $68 \%$ & $5 \%$ \\
Arrendamientos y cánones & $47 \%$ & $1 \%$ \\
Transportes & $5 \%$ & $0 \%$ \\
\hline
\end{tabular}

Fuente: Elaboración propia a partir de Martín-Cruz et al (2012) 
Con el fin de realizar la selección de inputs a utilizar en el análisis de la eficiencia, partimos del supuesto de que aquéllos utilizados por mayor cantidad de instrumentos dotan al DEA de una mayor capacidad de discriminación, al realizar una comparación más homogénea. Como puede observarse en la Tabla 5, los inputs utilizados en una mayor cantidad de instrumentos, son compras, sueldos y salarios y, comunicaciones y otros servicios, todos ellos empleados por más de un $90 \%$ de los instrumentos de la muestra. En conjunto, estos inputs representan un $91 \%$ del total de gasto ejecutado.

En resumen, la función de producción con la que se evaluará la eficiencia de los ICID incluye compras, sueldos y salarios $\mathrm{y}$, comunicaciones y otros servicios como inputs, y duración, actividades realizadas y áreas atendidas como outputs a maximizar. El análisis se realizará desde el lado de los outputs al asumirse que, con unos recursos dados (presupuesto disponible), cada instrumento intentará maximizar los resultados (llegar a una mayor cantidad de individuos o áreas, realizar más actividades, entre otros).

\section{Eficiencia de los instrumentos de cooperación internacional al desarrollo}

En este apartado se presentan los resultados obtenidos al aplicar el DEA, utilizando los outputs e inputs mencionados anteriormente, sobre una muestra de 72 ICID, finalmente reducida a 70 , una vez eliminados los instrumentos sin información para alguno de los outputs. Esta metodología requiere disponer de información completa sobre todas las variables de cada instrumento para poder efectuar su análisis.

En primer lugar, se analiza la eficiencia de los distintos ICID y, posteriormente, se analiza la eficiencia lograda por cada instrumento en función del país donde son ejecutados, para cada uno de los cuatro países objetivo prioritario de la ayuda al desarrollo española incluidos en la muestra. Tal como se explicó en el epígrafe anterior y por las razones expuestas, el modelo utilizado consta de tres outputs, duración, número de actividades realizadas y número de áreas de intervención, y tres inputs, compras, sueldos y salarios, comunicaciones y otros servicios.

En la Tabla 5 de los 70 instrumentos analizados solamente 18 son eficientes (alcanzan el valor 1), representando un $26 \%$ del total de la muestra. Este resultado pone de manifiesto el escaso número de ICID financiados, en los cuatro países analizados, que llegan a alcanzar la máxima eficiencia o, en otras palabras, llegan a ser plenamente eficientes. Este hecho, aunque pueda parecer sorprendente, en realidad, es el reflejo de los procedimientos de evaluación seguidos por la mayoría de las agencias de cooperación internacional para el desarrollo y, en particular, por la española. 


\section{Tabla 5}

\section{Eficiencia por tipo de Instrumento de Cooperación Internacional al Desarrollo}

\begin{tabular}{ccccc}
\hline Instrumento & $\begin{array}{c}\text { Total de } \\
\text { Instrumentos }\end{array}$ & $\begin{array}{c}\text { Instrumentos } \\
\text { Eficientes }\end{array}$ & $\begin{array}{c}\text { Eficiencia } \\
\text { Promedio }\end{array}$ & $\begin{array}{c}\text { Porcentaje } \\
\text { Eficientes }\end{array}$ \\
\hline $\begin{array}{c}\text { Ayudas de } \\
\text { Convocatoria } \\
\text { Abierta y } \\
\text { Permanente }\end{array}$ & 18 & 5 & 0,70 & $28 \%$ \\
$\begin{array}{c}\text { Subvenciones a } \\
\text { Organizaciones no } \\
\begin{array}{c}\text { Gubernamentales } \\
\text { para el Desarrollo }\end{array}\end{array}$ & 52 & 13 & 0,65 & $25 \%$ \\
TOTAL & 70 & 18 & 0,66 & $26 \%$ \\
\hline
\end{tabular}

Fuente: Elaboración propia a partir de los datos obtenidos de la Agencia Española de Cooperación Internacional para el Desarrollo (2009)

En concreto, las evaluaciones de los ICID se centran, básicamente, en la eficacia de las actuaciones y no en la eficiencia de las mismas. Por tanto, es lógico suponer un mayor interés de las autoridades por lograr elevados niveles de output sin tener en consideración el input utilizado o, dicho de otro modo, cuando las ONGD reciben un determinado nivel de input, no se llega a valorar 'ex-post' el output obtenido con amplio grado de detalle. La evaluación concreta de la eficiencia de cada proyecto financiado sería básica para lograr que ese porcentaje de ICID eficientes pudiera ser incrementado.

En cuanto a la eficiencia por tipo de instrumento, 5 CAP y 13 SO resultaron eficientes, lo que representa un $28 \%$ de las ayudas de convocatoria abierta permanente y un $25 \%$ de las subvenciones a ONGD. En cuanto a la eficiencia promedio de cada tipo de instrumento, los valores obtenidos son 0,70 para las CAP y 0,65 para las $\mathrm{SO}$, todos ellos en línea con los valores obtenidos por trabajos empíricos previos que analizaron el sector de la cooperación al desarrollo utilizando el DEA (Hernangómez-Barahona et al, 2006, 2007 y 2009; Martín-Pérez et al, 2005).

Este resultado, en el que se pone de manifiesto mayores niveles de eficiencia para las CAP frente a las SO, puede ser interpretado en dos sentidos. En el caso de las CAP, por una parte, las ONGD pueden tener muchos incentivos y compromiso con la unidad gestora encargada de adjudicar el proyecto y, por tanto, quieran alcanzar un alto nivel de eficiencia. Por otra 
parte, se podrían estar planteando objetivos muy ambiciosos para mostrar las capacidades del beneficiario al no existir un control tan exhaustivo como en el caso de las subvenciones a ONGD, al no comprobarse si todo lo planteado ha sido, efectivamente, llevado a cabo.

Lo anteriormente analizado permite comparar la eficiencia de los diferentes instrumentos, pero, también resulta de interés valorar los resultados para cada país concreto, a la hora de identificar si existe algún patrón que muestre diferencias significativas en función del país donde se ejecuta el instrumento, teniendo en cuenta sus características institucionales.

Como se desprende de los datos presentados en la Tabla 6 , el porcentaje de instrumentos eficientes por país es sumamente parecido (entre un 21\% y un $33 \%$ ), situándose alrededor del valor medio obtenido para el conjunto de la muestra (25\%). Este resultado hace del contexto en el que se ejecutan los ICID un factor poco relevante para conseguir un determinado nivel de eficiencia, con la excepción de Guatemala, país con un valor algo superior (33\%). En cuanto a la eficiencia promedio mostrada por los instrumentos analizados en cada uno de los países, los valores obtenidos para Ecuador, Guatemala y Mozambique son casi idénticos y muy parecidos al valor calculado para el conjunto de la muestra $(0,66)$, estando los resultados logrados por Marruecos ligeramente por encima del resto.

\section{Tabla 6}

\section{Eficiencia de los Instrumentos de Cooperación Internacional al Desarrollo por países}

\begin{tabular}{lcccc}
\hline \multicolumn{1}{c}{ País } & $\begin{array}{c}\text { Total de } \\
\text { Instrumentos }\end{array}$ & $\begin{array}{c}\text { Instrumentos } \\
\text { Eficientes }\end{array}$ & $\begin{array}{c}\text { Eficiencia } \\
\text { Promedio }\end{array}$ & $\begin{array}{c}\text { Porcentaje } \\
\text { Eficientes }\end{array}$ \\
\hline Ecuador & 13 & 3 & 0,62 & $23 \%$ \\
Guatemala & 9 & 3 & 0,62 & $33 \%$ \\
Marruecos & 30 & 8 & 0,70 & $26 \%$ \\
Mozambique & 19 & 4 & 0,63 & $21 \%$ \\
TOTAL & 70 & 18 & 0,66 & $25 \%$ \\
\hline
\end{tabular}

Fuente: Elaboración propia a partir de los datos obtenidos de la Agencia Española de Cooperación Internacional para el Desarrollo (2009)

Por tanto, a la hora de seleccionar países prioritarios de la ayuda, criterios como la inestabilidad política o económica no deberían ser elementos para apartar a un país de la recepción de fondos dirigidos a la cooperación, ya que parecen ser otras las circunstancias causantes de 
Eficiencia de las ayudas al desarrollo: El caso español

Martín-Pérez, Víctor; Martín-Cruz, Natalia y Serrano-Rodríguez, María José

la ineficiencia en la ejecución de los proyectos. Sería necesario considerar y estudiar otros factores explicativos de la falta de eficiencia como, por ejemplo, la motivación de las ONGD encargadas de llevar a cabo los proyectos o el conocimiento del entorno por parte de las organizaciones participantes en el proyecto, entre otras posibles razones.

En conclusión, de los resultados se deduce que Marruecos es el país donde los diferentes instrumentos de cooperación al desarrollo se ejecutan con mayores niveles de eficiencia promedio mientras Guatemala es la nación donde un mayor número de instrumentos se ejecutan de forma eficiente y logran un aprovechamiento óptimo de los recursos asignados para alcanzar sus objetivos.

En Guatemala, la puesta en marcha del programa de reactivación económica y social 2004-2005 "¡Vamos Guatemala!", puede ser el motivo detrás de los mejores resultados en este país, calculados en términos de proporción de proyectos ejecutados eficientemente.

Marruecos, por los intensos intercambios migratorios y comerciales con España, propicia un amplio conocimiento mutuo $\mathrm{y}$, por tanto, la posibilidad de alcanzar mayores niveles de eficiencia en los ICID ejecutados por ONGD españolas. A esto se debe añadir la existencia de la Comisión Mixta entre ambos países, mecanismo de concertación clave con las contrapartes marroquíes, así como las reuniones anuales de seguimiento de los proyectos, con un destacado papel en la consecución de proyectos eficientes.

Además de comparar los resultados por tipo de instrumento y país de ejecución, se puede reproducir este mismo análisis aplicándolo a un ámbito más reducido, como es cada uno de los cuatro países incluidos en la muestra considerados de forma independiente. De este modo, se puede realizar la comparación de instrumentos en un único entorno eliminando las diferencias cuyo origen pudiera venir dado por contextos distintos. Este análisis se efectuó para los instrumentos ejecutados en Marruecos, cuya muestra está formada por 30 ICID, único país donde, de forma autónoma, se puede realizar el análisis cumpliendo los requisitos exigidos por el DEA.

Tal y como puede observarse en la Tabla 7, donde se presenta el resumen de resultados del DEAaplicado a Marruecos, 6 CAP y 7 SO resultan eficientes, representando unos porcentajes, dentro de su correspondiente categoría, de un $55 \%$ y $37 \%$ respectivamente, en ambos casos superiores a los obtenidos para el total de la muestra, especialmente en el caso de las CAP. Estos valores se obtienen al aplicar el DEA sobre los 30 instrumentos ejecutados en Marruecos, no sobre toda la muestra, razón del cambio en el número de instrumentos que, finalmente, se muestran eficientes, incrementándose de 8 a 13.

En relación con los valores promedios, también son superiores a los conseguidos para toda la muestra. Como se expuso en el apartado explicativo de la metodología DEA, esta técnica pierde poder discriminante al reducirse el tamaño muestral, siendo la consecuencia no sólo un mayor número de observaciones plenamente eficientes sino también una eficiencia promedio más elevada para las restantes. 


\section{Tabla 7}

\section{Eficiencia por tipo de Instrumento de Cooperación Internacional al Desarrollo en Marruecos}

\begin{tabular}{ccccc}
\hline Instrumento & $\begin{array}{c}\text { Instrumentos } \\
\text { Totales }\end{array}$ & $\begin{array}{c}\text { Instrumentos } \\
\text { Eficientes }\end{array}$ & $\begin{array}{c}\text { Eficiencia } \\
\text { Promedio }\end{array}$ & $\begin{array}{c}\text { Porcentaje } \\
\text { Eficientes }\end{array}$ \\
\hline $\begin{array}{c}\text { Ayudas de } \\
\text { Convocatoria Abierta } \\
\text { y Permanente }\end{array}$ & 11 & 6 & 0,77 & $55 \%$ \\
$\begin{array}{c}\text { Subvenciones a } \\
\text { Organizaciones no } \\
\text { Gubernamentales } \\
\text { para el Desarrollo }\end{array}$ & 19 & 7 & 0,74 & $37 \%$ \\
\hline TOTAL & 30 & 13 & 0,75 & $43 \%$ \\
\hline
\end{tabular}

Fuente: Elaboración propia a partir de los datos obtenidos de la Agencia Española de Cooperación Internacional para el Desarrollo (2009)

Estos resultados son coherentes con los obtenidos cuando se aplica el análisis sobre los cuatro países, donde Marruecos es el país con mayores niveles de eficiencia promedio. Las CAP vuelven a ser el instrumento con los mejores niveles de eficiencia. Este ICID, por los dos motivos básicos ya comentados, permite a las ONGD alcanzar niveles mayores de eficiencia.

\section{Conclusiones}

La superioridad de las CAP, en términos de eficiencia, sobre las $\mathrm{SO}$ en todos los países analizados. A la luz de este resultado, la eficiencia lograda parece tener cierta independencia del entorno concreto donde se ejecutan las actuaciones y depender, en mayor medida, del tipo de instrumento de cooperación a través del cual se haya canalizado la ayuda.

Una explicación de este resultado puede venir dada por la forma de concesión de los instrumentos, mucho más exigente en el caso de las CAP. Esto conlleva que sólo las mejores ONGD puedan verse recompensadas con un instrumento de este tipo, repercutiendo en gran medida sobre su reputación. Se genera, así, un efecto de retroalimentación, cuya consecuencia es incentivar a las ONGD a ser más eficaces y eficientes para poder seguir optando a la concesión de este tipo instrumento y, de este modo, mantener el potente efecto señalizador enviado a otros potenciales donantes, especialmente los grandes donantes institucionales privados, sobre el buen hacer de la entidad.

Otra posible explicación puede venir dada por el tamaño (presupuesto) de cada tipo de instrumento. Generalmente, el tamaño de las CAP es inferior al de las SO, traduciéndose en unos objetivos menos ambiciosos y, quizá, más precisos por comparación con las SO, cuyos proyectos/programas, en ocasiones, tienden a querer abarcar demasiadas metas, haciendo más difícil su consecución. 
Eficiencia de las ayudas al desarrollo: El caso español Martín-Pérez, Víctor; Martín-Cruz, Natalia y Serrano-Rodríguez, María José

Los resultados por país también ponen de relieve la importancia de centrarse en ámbitos de actuación más reducidos. Así, Guatemala, país donde se ejecutan proyectos con un menor nivel de heterogeneidad, presenta un mayor porcentaje de instrumentos eficientes. En la línea con lo postulado por las Declaraciones de París y Accra, las agencias que en cada país se encargan de planificar y ejecutar la ayuda al desarrollo, deberían centrarse en los ámbitos/sectores donde cuentan con mayor experiencia y competencia, dejando los demás para las agencias de otros países puesto que la proliferación de actuaciones en ámbitos diversos da lugar a una dispersión de esfuerzos cuya consecuencia es la penalización en los resultados alcanzados.

En cualquier caso, es preciso recalcar el amplio margen de mejora existente tanto para las CAP como para las SO, como reflejan los resultados obtenidos donde se observa que menos de un $30 \%$ de los instrumentos ejecutados logran el nivel de eficiencia máximo alcanzable. Este hecho debe hacer reflexionar sobre si los instrumentos utilizados para canalizar la ayuda al desarrollo están fijando unos objetivos demasiado ambiciosos (en la práctica, imposibles de cumplir) así como si los actores encargados de ejecutarlos están debidamente capacitados para las actuaciones a realizar.

También debe considerarse si la eficiencia no es todavía una magnitud relevante en los instrumentos de ejecución de la ayuda al desarrollo, guiándose exclusivamente por la eficacia. Directamente relacionada con esta última afirmación, debe analizarse si los controles aplicados por los donantes siguen siendo mayoritariamente ex-ante (previos a la concesión del instrumento) dejando de lado los controles expost (cuando finaliza la ejecución del instrumento) por la dificultad y el coste derivados de su implementación.

Las implicaciones derivadas de estos resultados para la AECID, como financiador de los instrumentos analizados, son evidentes, en particular, el uso de esta medida de eficiencia con carácter ex-post, permitiría valorar, de forma concreta y específica, los resultados alcanzados por cada proyecto y plantear, de cara al futuro, acciones correctoras, así como, servir de criterio de selección ex-ante de futuros ICID. Igualmente, la comparación entre instrumentos da la oportunidad de reconocer en qué condiciones un ICID podría permitir alcanzar mayores niveles de eficiencia.

Sería deseable poder contar con un mayor número de observaciones e, incluso, diversidad de instrumentos. Sin embargo, resulta sumamente complicado obtener información sistematizada de los inputs y outputs de los ICID debido a la falta de un procedimiento común e informatizado de recogida de la información por parte del organismo encargado, en este caso, la AECID.

Podría ser de interés ampliar el periodo de estudio (5 años) y, adicionalmente, plantear un análisis por anualidades para valorar si la composición del comité de selección influye sobre la elección de proyectos más o menos eficientes. También sería deseable disponer de alguna medida de impacto de los instrumentos que permita mejorar la calidad de los inputs y outputs utilizados para caracterizar la función de producción. 


\section{Referencias bibliográficas}

Alesina, Alberto y Dollar, David (2000), Who gives foreign aid to whom and why? Journal of Economic Growth, Volumen 5 , № 1 , The Netherlands, Springer, pp 33-63.

Al-Shammari, Minwir (1999), A multi-criteria data envelopment analysis model for measuring the productive efficiency of hospitals. International Journal of Operations and Production Management, Volumen 19, $\mathrm{N}^{\circ} 9$, England, Emerald, pp 879-890.

Andrés-Alonso, Pablo; Martín-Cruz, Natalia y Romero-Merino, Elena (2006), The governance of nonprofit organizations: Empirical evidence from nongovernmental development organizations in Spain. Nonprofit and Voluntary Sector Quarterly, Volumen $35, N^{\circ} 4$, USA, Sage, pp 588-604.

Andrés-Alonso, Pablo; Azofra-Palenzuela, Valentín y Romero-Merino, Elena (2010), Beyond the disciplinary role of governance: How boards add value to Spanish foundations. British Journal of Management, Volumen 21, $\mathrm{N}^{\circ} 1$, England, WileyBlackwell, pp 100-114.

Avkiran, Necmi (2001), Investigating technical and scale efficiencies of Australian Universities through data envelopment analysis. SocioEconomic Planning Sciences, Vol. 35, no. 1, United Kingdom, Pergamon Elsevier Science, pp 57-80.

Baber, William R; Roberts, Andrea A. y Visvanathan, Gnanakumar (2001), Charitable organizations' strategies and program-spending ratios. Accounting Horizons, Volumen $15, N^{\circ} 4$, USA, American Accounting Association, pp 329-343.

Bandyopadhyay, Subhayu y Wall, Howard J. (2007), The determinants of aid in the post cold-war era. En Sajal Lahiri (Ed.) Theory and Practice of
Foreign Aid (Frontiers of Economics and Globalization, Vol. 1). The Netherlands, Emerald, Chapter 19, pp 387-402.

Banerjee, Abhijit V. (2009), Big answers for big questions: The presumption of growth policy. En Jessica Cohen y William Easterly (Eds.) What Works in Development? Thinking Big and Thinking Small. Washington: The Brooking Institution, pp 207-221.

Banker, Rajiv D.; Charnes, Abraham and Cooper, William W. (1984), Some models for estimating technical and scale efficiencies in data envelopment analysis. Management Science, Volumen 30 , No 9, USA, Informs, pp 10781092.

Banker, Rajiv D.; Janakiraman, Surya y Natarajan, Ram (2004), Analysis of trends in technical and allocative efficiency: An application to Texas public school districts. European Journal of Operational Research, Volumen 145, № 2, The Netherlands, Elsevier Science, pp 477-491.

Boone, Peter (1996), Politics and effectiveness of foreign aid. European Economic Review, Volumen 40, $\mathrm{N}^{\circ} 2$, The Netherlands, Elsevier Science, pp 289-329.

Callen, Jeffrey y Frank, Haim (1993), Agency and efficiency in nonprofit organizations: The case of "specific health focus" charities. The Accounting Review, Volumen 68 , $\mathrm{N}^{\circ}$ 1, USA, American Accounting Association, pp 48-65.

Callen, Jeffrey L. (1994), Money donations, volunteering and organizational efficiency. Journal of Productivity Analysis, Volumen $5, \mathrm{~N}^{\circ} 3$, The Netherlands, Springer, pp 215-228.

Charnes, Abraham; Cooper, William W. y Rhodes, Edwardo (1978), Measuring the efficiency of decisions making units. European 
Journal of Operational Research, Volumen 2, № 6, The Netherlands, Elsevier Science, pp 429-444.

Charnes, Abraham; Cooper, William W.; Lewin, Arie Y. y Seiford, Lawrence M. (eds.) (1994), Data envelopment analysis. Theory, methodology and applications. USA, Boston, MA: Kluwer Academic Publishers.

Dowling, John M. y Hiemenz, Ulrich (1985), Biases in allocation of foreign aid: Some new evidence. World Development, Volumen 13, $\mathrm{N}^{\circ}$ 4, England, Elsevier Science, pp 535-541.

Duflo, Esther y Kremer, Michael (2003), Use of randomization in the evaluation of development effectiveness. World Bank Operations Evaluation Department (OED) Conference on Evaluation and Development Effectiveness, USA, Washington.

Farrell, Michael J. (1957), The measurement of productive efficiency. Journal of the Royal Statistical Society, Volumen $120, \mathrm{~N}^{\circ} 3$, United Kingdom, Willey-Blackwell, pp 253-290.

Farrell, Michael J. y Fieldhouse, Mark (1962), Estimating efficient production functions under increasing returns to scale. Journal of the Royal Statistical Society, Volumen 125, $\mathrm{N}^{\circ}$ 2, United Kingdom, WilleyBlackwell, pp 252-267.

García, Florencio; Marcuello, Carmen; Serrano, Diana y Urbina, Olga (1999), Evaluation of efficiency in primary health care centres: An application of data envelopment analysis. Financial, Accountability and Management, Volumen $15, \mathrm{~N}^{\circ}$ 1, United Kingdom, Wiley-Blackwell, pp 67-83.

García, Lucía y Marcuello, Carmen (2007), Eficiencia y captación de fondos en las organizaciones no gubernamentales para el desarrollo. CIRIEC-España, Revista de Economía Pública, Social y Cooperativa, Volumen
58, España, Centro Internacional de Investigación e Información sobre la Economía Pública, Social y Cooperativa, pp 221-249

Greenlee, Janet y Brown, Karen (1999), The impact of accounting information on contributions to charitable organizations. Research in Accounting Regulation, Volumen 13, England, Elsevier Science, pp 111-125.

Hernangómez-Barahona, Juan; Martín-Cruz, Natalia y Martín-Pérez, Víctor (2006), La relevancia del objetivo en la medida de la eficiencia. Un análisis para las ONGD españolas desde la teoría del comportamiento. Boletín Económico del ICE, Volumen 2884, España, Ministerio de Economía y Competitividad, pp 17-30.

Hernangómez-Barahona, Juan; Martín-Cruz, Natalia y Martín-Pérez, Víctor (2007), El deleite de la eficiencia. Universia Business Review, Volumen 14, España, Universia, pp 56-67.

Hernangómez-Barahona, Juan; MartínPérez, Víctor y Martín-Cruz, Natalia (2009), Implicaciones de la organización interna sobre la eficiencia. La aplicación de la teoría de la agencia y la metodología DEA a las ONGD españolas. Cuadernos de Economía y Dirección de la Empresa, Volumen 40, España, Elsevier Doyma, pp 17-49.

Hofmarcher, Maria; Paterson, lain y Riedel, Monica (2002), Measuring hospital efficiency in Austria: A DEA approach. Health Care Management Science, Volumen 5, № 1, USA, Springer, pp 7-14.

Hyndman, Noel (1991), Contributions to charities. A comparison of their information needs and the perceptions of such by the providers of information. Financial Accountability and Management, Volumen $7, \mathrm{~N}^{\circ} 2$, United Kingdom, Willey-Blackwell, pp 69-82. 
Isenman, Paul (1976), Biases in aid allocation against poorer and larger countries. World Development, Volumen 4, $N^{\circ}$ 8, England, Elsevier Science, pp 631-641.

Johnes, Jill y Johnes, Geraint (1995), Research funding and performance in U.K. university departments of economics: A frontier analysis. Economics of Education Review, Volumen 14, No 3, USA, Pergamon Elsevier Science, pp 301-314.

Jordan, Lisa y Van Tuijl, Peter (Eds.) (2006), NGO accountability: Politics, principles and innovations. United Kingdom, London: Earthscan.

Khumawala, Saleha B. y Gordon, Teresa P. (1997), Bridging the credibility of GAAP: Individual donors and the new accounting standards for nonprofit organizations. Accounting Horizons, Volumen $11, N^{\circ} 3$, USA, American Accounting Association, pp 45-68.

Korhonen, Pekka; Tainio, Risto y Wallenius, Jyrki. (2001), Value efficiency analysis of academic research. European Journal of Operational Research, Volumen 130, $N^{\circ} 1$, The Netherlands, Elsevier Science, pp 121-132.

Kuziemko, llyana y Werker, Eric (2006), How much is a seat on the security council worth? Foreign aid and bribery at the United Nations. Journal of Political Economy, Volumen 114, № 5 , USA, University of Chicago Press, pp 905-930.

Ley, Eduardo (1991), Eficiencia productiva: Un estudio aplicado al sector hospitalario. Investigaciones Económicas, Volumen 15, $\mathrm{N}^{\circ} 1$, España, Fundación SEPI, pp 7188.

Magnussen, Jon (1996), Efficiency measurement and the operationalization of hospital production. Health Services Research, Volumen $31, N^{\circ} 1$, USA, Wiley-Blackwell, pp 21-37
Maizels, Alfred y Nissanke, Machiko (1984), Motivations for aid to developing countries. World Development, Volumen 12, $\mathrm{N}^{\circ}$ 9, England, Elsevier Science, pp 879-900.

Marcuello, Carmen (1999), Análisis de la conducta y eficiencia de las organizaciones nogubernamentales para el desarrollo españolas. Información Comercial Española, Volumen 778, mayo-junio, España, Ministerio de Industria, Turismo y Comercio, pp 181-196.

Martín-Pérez, Víctor; Martín-Cruz, Natalia y Hernangómez-Barahona, Juan (2005), La valoración de los resultados de las entidades sin fines de lucro. Una aplicación en el análisis de la eficiencia de la arquitectura organizativa de las ONGD españolas. Revista AECA Volumen 71, España, Asociación Española de Contabilidad y Administración de Empresas, pp 34-40.

Martín-Cruz, Natalia; Martín-Pérez, Víctor y Gámez-Alcalde, César (2012), La eficiencia y el «misreporting) contable en las ONGD españolas. Análisis de proyectos de cooperación internacional para el desarrollo. Academia, Revista Latinoamericana de Administración, Volumen 51, No 4, Colombia, Consejo Latinoamericano de Escuelas de Administración, pp 1-14.

Martínez, Marcelino (2003), La medición de la eficiencia en las instituciones de educación superior. Bilbao, España: Fundación BBVA.

McKinley, Robert D. y Little, Richard (1977), A foreign policy model of U.S. aid allocation. World Politics, Volumen 30, № 1, USA, Cambridge University Press, pp 58-86.

McKinley, Robert D. y Little, Richard (1979), The U.S. aid relationship: A test of the recipient need and the donor interest models. Political Studies, Volumen 27, $\mathrm{N}^{\circ} 2$, England, WileyBlackwell, pp 236-250. 
McMillan, Melville y Datta, Debasish (1998), The relative efficiencies of Canadian universities: A DEA perspective. Canadian Public Policy, Volumen 24, $\mathrm{N}^{\circ} 4$, Canada, University of Toronto Press, pp 485-511.

Meyer, Carrie A. (1995), Opportunism and NGOs: Entrepreneurship and green north-south transfers. World Development, Volumen 23, $\mathrm{N}^{\circ}$ 8, England, Elsevier Science, pp 1277-1289.

Naciones Unidas (2005), Informe sobre desarrollo humano 2005: La cooperación al desarrollo ante una encrucijada. Ayuda al desarrollo, comercio y seguridad en un mundo desigual. Madrid, España: Ediciones Mundi-Prensa.

Neumayer, Eric (2003a), The determinants of aid allocation by regional multilateral development banks and United Nations agencies. International Studies Quarterly, Volumen 47, USA, Wiley-Blackwell, pp 101-122.

Neumayer, Eric (2003b), Is respect for human rights rewarded? An analysis of total bilateral and multilateral aid flows. Human Rights Quarterly, Volumen 25, No 2, USA, Johns Hopkins University Press, pp 510-527.

O’Brien, Finbar (2009), Preface by UNICEF Director of Evaluation Office. En Marco Segone (Ed.) Country-led monitoring and evaluation systems. Better evidence, better policies, better development results. Suiza, UNICEF, pp 2-3.

OCDE (2005). Declaración de París sobre la eficacia de la ayuda al desarrollo y programa de acción de Accra. Organización para la Cooperación y el Desarrollo Económico, marzo [consulta: 20 de mayo de 2011]. Disponible en http://www.oecd.org/development/ ffectiveness/34580968.pdf

OCDE (2008a). The Accra high level forum (HLF3) and the Accra agenda for action. Organización para la Cooperación y el
Desarrollo Económico, septiembre [consulta: 22 de mayo de 2011]. Disponible en http://www. oecd.org/dac/effectiveness/ eaccraagendaforaction.htm

OCDE (2008b). Is it ODA? Factsheet. Organización para la Cooperación y el Desarrollo Económico, noviembre [consulta: 22 de mayo de 2011]. Disponible en http://www. oecd.org/aoecd/21/21/34086975. pdf. Fecha de consulta:

OCDE (2011). The Busan partnership for effective development cooperation. [consulta: 24 de mayo de 2011]. Disponible en http:// www.oecd.org/dac/effectiveness/ busanpartnership.htm

Parsons, Linda M. (2003), Is accounting information from nonprofit organizations useful to donors? A review of charitable giving and valuerelevance. Journal of Accounting Literature, Volumen 22, USA, University of Florida, pp 104-129.

Posnett, John y Sandler, Todd (1989), Demand for charity donations in private nonprofit markets. The case of the U.K. Journal of Public Economics, Volumen $40, \mathrm{~N}^{\circ} 2$, The Netherlands, Elsevier Science, pp 187-200.

Ram, Rati (2003), Roles of bilateral and multilateral aid in economic growth of developing countries. KYKLOS, Volumen 56, $\mathrm{N}^{\circ} 1$, England, WileyBlackwell, pp 95-110.

Roberts, Andrea A; Smith, Pamela y Taranto, Karen (2004), Marginal spending and efficiency in charities. Proceedings of the Annual American Accounting Association Meeting, Orlando, Florida.

Ruggiero, John (1999), Nonparametric analysis of educational costs. European Journal of Operational Research, Volumen 119, No. 3, The Netherlands, Elsevier Science, pp. 605-612.

Svensson, Jakob (1999), Aid, growth and democracy. Economics and Politics, 
Volumen 11, № 3, USA, Wiley, pp. 275-297.

Tinkelman, Daniel (1998), Differences in sensitivity of financial statement users to joint cost allocations: The case of nonprofit organizations. Journal of Accounting, Auditing and Finance, Volumen $13, \mathrm{~N}^{\circ} 4$, USA, Sage, pp 377-393.

Trumbull, William N. y Wall, Howard J. (1994), Estimating aid allocation criteria with panel data. Economic Journal, Volumen 104, № 425, England, Wiley-Blackwell, pp 876-882.

Valdmanis, Vivian (1992), Sensitivity analysis for DEA models. An empirical example using public vs. NFP hospitals. Journal of Public Economics, Volumen 48, № 2, The Netherlands, Elsevier Science, pp 185-205.

Wall, Howard J. (1995), The allocation of official development assistance. Journal of Policy Modeling,
Volumen 17, № 3, USA, Elsevier Science, pp 307-314.

Watcharasriroj, Budsakorn y Tang, John (2004), The effects of size and information technology on hospital efficiency. Journal of Hich Technology Management Research, Volumen 15, $\mathrm{N}^{\circ} 1$, United Kingdom, Elsevier Science, pp 1-16.

Weisbrod, Burton A. y Dominguez, Nestor D. (1986), Demand for collective goods in private nonprofit markets: Can fundraising expenditures help overcome free-rider behavior? Journal of Public Economics, Volumen $30, \mathrm{~N}^{\circ} 1$, The Netherlands, Elsevier Science, pp 83-96.

Younas, Javed (2008), Motivation for bilateral aid allocation: Altruism or trade benefits. European Journal of Political Economy, Volumen 24, $\mathrm{N}^{\circ} 3$, The Netherlands, Elsevier Science, pp 661-674. 